SLAC-PUB-8002

November, 1998

\title{
Collider Signatures of New Large Space Dimensions
}

\author{
Eugene A. Mirabelli, Maxim Perelstein, and Michael E. Peskin円 \\ Stanford Linear Accelerator Center \\ Stanford University, Stanford, California 94309 USA
}

\begin{abstract}
Recently, Arkani-Hamed, Dimopoulos, and Dvali have proposed that there are extra compact dimensions of space, accessible to gravity but not to ordinary matter, which could be macroscopically large. In this letter, we argue that high-energy collider processes in which gravitons are radiated into these new dimensions place significant, model-independent constraints on this picture. We present the constraints from anomalous single photon production at $e^{+} e^{-}$colliders and from monojet production at hadron colliders.
\end{abstract}

Submitted to Physical Review Letters

\footnotetext{
${ }^{1}$ Work supported by the Department of Energy, contract DE-AC03-76SF00515.
} 
The Standard Model of strong, weak, and electromagnetic interactions has been dramatically successful in explaining the rates of high-energy $e^{+} e^{-}$and $p \bar{p}$ reactions and the properties of the $W$ and $Z$ bosons. This great success, however, has focused attention on the fact the Standard Model (SM) requires a number of choices for its input parameters which are very difficult to understand. Among these are the value of the Higgs boson mass parameter $\mu^{2}$ and the value of the cosmological constant $\lambda$. If one assumes that the most fundamental scale in Nature is the Planck scale, $M_{\mathrm{Pl}}=G_{N}^{-1 / 2}=10^{19} \mathrm{GeV}$ and writes these parameters in terms of this scale, one finds $\mu^{2} \sim 10^{-34} M_{\mathrm{Pl}}^{2}, \lambda \sim 10^{-116} M_{\mathrm{Pl}}^{4}$.

The mystery of these small parameters has motivated many authors to consider radical ideas for the manner in which gravity is unified with the other fundamental interactions. The introduction of supersymmetry can lower the natural mass scale for $\mu^{2}$ and $\lambda$ to $1 \mathrm{TeV}$. This ameliorates the problem of the Higgs mass but is not nearly enough of a reduction to solve the cosmological constant problem. Many authors have investigated whether a string theory of quantum gravity can provide a further reduction. String theory includes the possibility of additional microscopic space dimensions. In this context, Antoniadis [1] has proposed that Nature may contain additional compact dimensions of size $\hbar / \mathrm{TeV}$ [2].

Recently, several groups [3, 4, 5] have extended this proposal using new ideas about the strong-coupling behavior of string theory. In this regime, string theory may contain solitons or mirror surfaces that occupy lower-dimensional hypersurfaces, with some species of particle restricted to these objects. One can then imagine that the quarks, leptons, and gauge bosons of the SM live on a 4-dimensional hypersurface inside the full space-time, while gravity lives in the full, higher-dimensional space. Arkani-Hamed, Dimopoulos, and Dvali (ADD) [4] have argued that, in these models, the fundamental gravitational scale can be as low as TeV energies, while the size of the extra dimensions can be as large as a millimeter.

If indeed gravity becomes strong at $\mathrm{TeV}$ energies, gravitons should be radiated at significant rates in high-energy particle collisions. In collider experiments, higher-dimensional gravitons $(\mathrm{G})$ appear as massive spin-2 neutral particles which are not observed by collider detectors. As ADD pointed out, $\mathrm{G}$ radiation leads to missing-energy signatures in which a photon or a jet is produced with no observable particle balancing its transverse momentum. In this paper, we compute the rates of the missing-energy processes

$$
e^{+} e^{-} \rightarrow \gamma+(\text { missing }), \quad p \bar{p} \rightarrow \text { jet }+(\text { missing })
$$

and the corresponding experimental constraints. We show that these experiments actually give the strongest present constraints on the size of the extra dimensions, and that future experiments will have even better sensitivity.

Conventions. In this paper, we assume that the gravitational field is the only field that propagates in the extra dimensions. It is likely that, in realistic models, the extra dimensions will also contain scalar, vector, and even fermion fields that couple to the SM particles with gravitational strength. These particles would produce additional, model-dependent, missingenergy signatures beyond those we consider here. We also assume that the typical momenta with which gravitons are emitted are small relative to the thickness of the hypersurface on 
which the SM particles live, and also relative to the fundamental gravitational scale. In this limit, the higher-dimensional gravitational field couples to the energy-momentum tensor of the SM, with precisely the coupling of standard 4-dimensional gravity [6]. To compute the rate of emission of a single $\mathrm{G}$ particle, we interpret the $\mathrm{G}$ momentum in the extra dimensions as a 4 -dimensional mass for this spin-2 particle and use the Lagrangian

$$
\delta \mathcal{L}=-\left(8 \pi G_{N}\right) G_{\mu \nu} T^{\mu \nu}
$$

Under our assumptions, this coupling is model-independent.

For definiteness, we will assume that there are $n$ extra dimensions, and that these are compactified on an $n$-dimensional torus of periodicity $2 \pi R$. With our choices, the gravitational potential for $r$ just larger than $R$ takes the form

$$
V(r)=-\frac{G_{N} m_{1} m_{2}}{r}\left(1+2 n e^{-r / R}+\cdots\right) .
$$

Macroscopic measurements of the force of gravity constrain $R$ to be less than a millimeter; for example, for $n=2, R<0.77 \mathrm{~mm}$ at $95 \%$ confidence [7] 8]. For $r \ll R$, the potential goes over to the $r^{-(n+1)}$ dependence characteristic of the higher-dimensional space. The coefficient of this potential is proportional to a power of fundamental gravitational scale. ADD define this scale by the formula

$$
M^{n+2} R^{n}=\left(4 \pi G_{N}\right)^{-1}
$$

With this definition, $M=1 \mathrm{TeV}$ corresponds to $R=0.68 \mathrm{~mm}$ for $n=2$ and to $R=$ $3.0 \times 10^{-12} \mathrm{~cm}$ for $n=6$.

In computing the emission of $\mathrm{G}$ particles, it is necessary to sum over the possible values of the higher-dimensional momenta $k_{\perp}$. This is, equivalently, a sum or integral over values of the 4-dimensional mass

$$
\sum_{k_{\perp}}=R^{n} \int d^{n} m=\frac{1}{2} \Omega_{n} R^{n} \int\left(m^{2}\right)^{(n-2) / 2} d m^{2}=\frac{\Omega_{n}}{8 \pi} M^{-(n+2)} \int\left(m^{2}\right)^{(n-2) / 2} d m^{2} G_{N}^{-1},
$$

where $\Omega_{n}$ is the volume of the unit sphere in $n$ dimensions $(=2 \pi$ for $n=2)$.

Electron-positron collisions. With these conventions, it is now straightforward to compute the rate of $e^{+} e^{-}$annihilation into an anomalous single photon recoiling against an unobserved $\mathrm{G}$. This reaction could potentially be observed at the CERN $e^{+} e^{-}$collider LEP 2, or at a higher-energy $e^{+} e^{-}$collider.

The differential cross sections for the reactions $e_{L}^{-} e_{R}^{+} \rightarrow \gamma G$, considered in the center of mass system for a $\mathrm{G}$ of mass $m$, is given by [9]

$$
\begin{aligned}
& \frac{d \sigma}{d \cos \theta}=\frac{\pi \alpha G_{N}}{1-m^{2} / s} {\left[\left(1+\cos ^{2} \theta\right)\left(1+\left(\frac{m^{2}}{s}\right)^{4}\right)\right.} \\
&\left.+\left(\frac{1-3 \cos ^{2} \theta+4 \cos ^{4} \theta}{1-\cos ^{2} \theta}\right) \frac{m^{2}}{s}\left(1+\left(\frac{m^{2}}{s}\right)^{2}\right)+6 \cos ^{2} \theta\left(\frac{m^{2}}{s}\right)^{2}\right]
\end{aligned}
$$




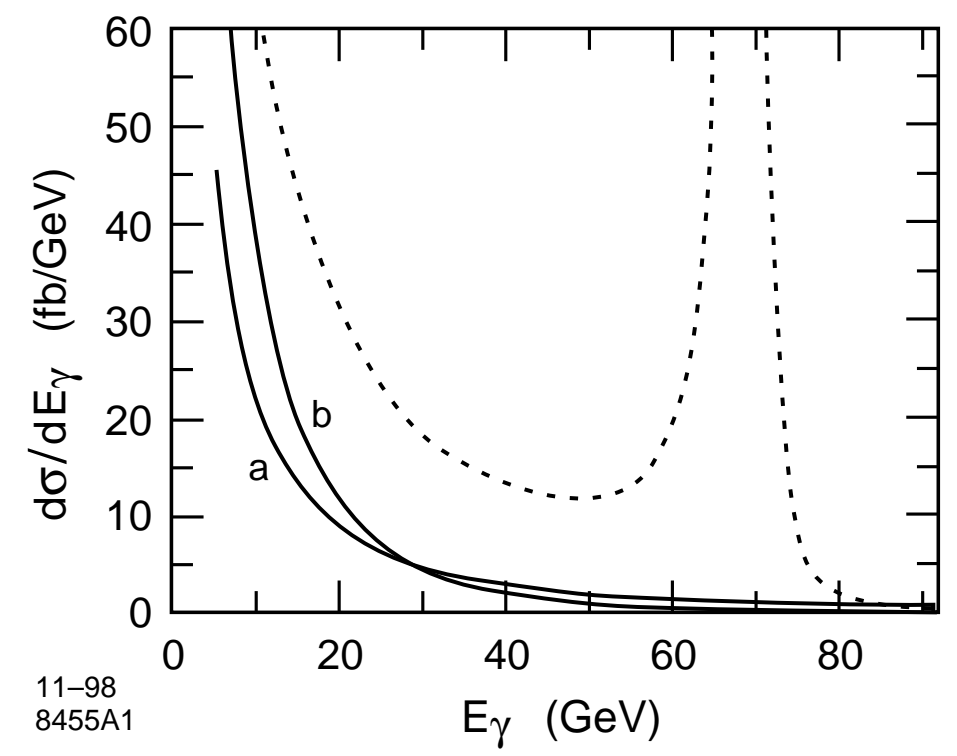

Figure 1: Energy spectrum of single photons recoiling against higher-dimensional gravitons $\mathrm{G}$, computed for $e^{+} e^{-}$collisions at $\sqrt{s}=183 \mathrm{GeV}$ with an angular cut $|\cos \theta|<0.95$. The dotted curve is the Standard Model expectation. The solid curves show the additional cross section expected in the model of ref. [4] with (a) $n=2, M=1200 \mathrm{GeV}$, (b) $n=6, M=520$ $\mathrm{GeV}$.

The same formula holds for $e_{R}^{-} e_{L}^{+}$; the helicity-violating cross sections are zero. These expressions must be integrated over the phase space (5). The cross section behaves as $\sigma \sim s^{n / 2} / M^{n+2}$. Thus, the production of anomalous single photons increases dramatically as the center-of-mass energy is raised.

In the SM, single photon events are produced in the reaction $e^{+} e^{-} \rightarrow \gamma \nu \bar{\nu}$, which can proceed through $s$-channel $Z^{0}$ exchange or (for the case of $\nu_{e}$ ) through $t$-channel $W$ exchange [10]. The effect of $G$ emission would be observable as an enhancement of the cross section for single- $\gamma$ production above that of this SM source. The single- $\gamma$ cross section has been measured at LEP 2 at $\sqrt{s}=183 \mathrm{GeV}$ by the ALEPH [11 and OPAL 12 experiments. The measurements agree with the SM prediction to $6 \%$ accuracy. If we integrate our prediction for the $\mathrm{G}$ signal over the kinematic region studied in these experiments, we find, for the case $n=2$, the limits $R<0.48 \mathrm{~mm}, M>1200 \mathrm{GeV}$ at $95 \%$ confidence. Limits for higher values of $n$ are given in Table 1 .

In Figure 1, we show the energy distribution of single photons recoiling against $\mathrm{G}$ particles for the cases $n=2$ and $n=6$, for the parameter values at our limit, compared to the singlephoton distribution from the SM process. The peak in the SM cross section results from the process in which the $\gamma$ recoils against an on-shell $Z^{0}$ which decays invisibly. Some additional advantage can be gained, then, in applying a cut which excludes this peak. For the kinematic 
region $20<E_{\gamma}<50 \mathrm{GeV},\left|\cos \theta_{\gamma}\right|<0.95$ and $\sqrt{s}=183 \mathrm{GeV}$, we find the cross section for $\mathrm{G}$ production

$$
\sigma=630 / M^{4}, 46 / M^{6}, 1.8 / M^{8} \mathrm{fb},
$$

for $n=2,4,6$ and $M$ in $\mathrm{TeV}$.

Higher-energy studies of $e^{+} e^{-}$annihilation will be done at a linear $e^{+} e^{-}$collider (LC). We have already noted that higher energy alone should lead to much higher sensitivity to $\mathrm{G}$ production. But the LC also offers another advantage, the possibility of electron beam polarization, which can be used to suppress the dominant $t$-channel $W$ exchange piece of the SM background process. At $\sqrt{s}=1 \mathrm{TeV}$, with electron polarization $P=+0.9$ (righthanded), integrating over the kinematic region $50 \mathrm{GeV}<E_{\gamma}<400 \mathrm{GeV},\left|\cos \theta_{\gamma}\right|<0.95$, we find a SM background cross section of $82 \mathrm{fb}$ and a $\mathrm{G}$ signal cross section of

$$
\sigma=20 / M^{4}, 46 / M^{6}, 55 / M^{8} \mathrm{pb}
$$

for $n=2,4,6$ and $M$ in $\mathrm{TeV}$. To quantify the effect of this measurement, we assume that this cross section can be measured with $5 \%$ accuracy, and that the value to be found agrees with the SM. Then the measurement would give very strong limits on $R$ and $M$ which are listed in Table 1.

Proton-antiproton collisions. In a similar way, proton-antiproton collisions can lead to processes in which a single parton is produced at large transverse momentum recoiling against a $\mathrm{G}$ particle. This leads to a monojet signature of $\mathrm{G}$ production - a jet plus missing transverse energy $\left(E_{T}\right)$ - which may be visible at the Fermilab Tevatron collider. The search for this reaction complements the search in $e^{+} e^{-}$reactions in the familiar way, with the higher energy available in hadron collisions compensating important losses in the definition of the signal.

The production of jets with large $E_{T}$ recoiling against $G$ particles can arise from the parton subprocesses $q \bar{q} \rightarrow G g, q g \rightarrow q G, \bar{q} g \rightarrow \bar{q} G$, and $g g \rightarrow g G$. The polarization- and color-averaged cross section for $q \bar{q} \rightarrow g G$ can be obtained directly from Eq. (6)

$$
\begin{aligned}
\frac{d \sigma}{d \cos \theta} & =\frac{2}{9} \frac{\pi \alpha_{s} G_{N}}{1-m^{2} / s}\left[\left(2-4 \frac{4 u t}{\left(s-m^{2}\right)^{2}}\right)\left(1+\left(\frac{m^{2}}{s}\right)^{4}\right)\right. \\
+ & \left.\left(2 \frac{\left(s-m^{2}\right)^{2}}{4 u t}-5+4 \frac{4 u t}{\left(s-m^{2}\right)^{2}}\right) \frac{m^{2}}{s}\left(1+\left(\frac{m^{2}}{s}\right)^{2}\right)+6\left(\frac{u-t}{s-m^{2}}\right)^{2}\left(\frac{m^{2}}{s}\right)^{2}\right]
\end{aligned}
$$

where $s, t, u$ are the Mandelstam variables: $t, u=-\frac{1}{2} s\left(1-m^{2} / s\right)(1 \mp \cos \theta)$. The cross section for $q g \rightarrow q G$ can be obtained from this expression by crossing $s \leftrightarrow t$ :

$$
\begin{aligned}
\frac{d \sigma}{d \cos \theta} & =\frac{\pi \alpha_{s} G_{N}(-t / s)\left(1-m^{2} / s\right)}{12\left(1-m^{2} / t\right)^{2}}\left[\left(2-4 \frac{4 u s}{\left(t-m^{2}\right)^{2}}\right)\left(1+\left(\frac{m^{2}}{t}\right)^{4}\right)\right. \\
+ & \left.\left(2 \frac{\left(t-m^{2}\right)^{2}}{4 u s}-5+4 \frac{4 u s}{\left(t-m^{2}\right)^{2}}\right) \frac{m^{2}}{t}\left(1+\left(\frac{m^{2}}{t}\right)^{2}\right)+6\left(\frac{s-u}{t-m^{2}}\right)^{2}\left(\frac{m^{2}}{t}\right)^{2}\right] .
\end{aligned}
$$


The cross section for $\bar{q} g \rightarrow \bar{q} G$ is also given by (10). For the process $g g \rightarrow g G$, we find the polarization- and color-averaged cross section [13]

$$
\begin{aligned}
\frac{d \sigma}{d \cos \theta}= & \frac{3}{16} \frac{\pi \alpha_{s} G_{N}}{\left(1-m^{2} / s\right)\left(1-\cos ^{2} \theta\right)}\left[\left(3+\cos ^{2} \theta\right)^{2}\left(1+\left(\frac{m^{2}}{s}\right)^{4}\right)\right. \\
& \left.-4\left(7+\cos ^{4} \theta\right) \frac{m^{2}}{s}\left(1+\left(\frac{m^{2}}{s}\right)^{2}\right)+6\left(9-2 \cos ^{2} \theta+\cos ^{4} \theta\right)\left(\frac{m^{2}}{s}\right)^{2}\right] .
\end{aligned}
$$

All of these formulae must be integrated over the G mass spectrum using the measure (5). The rate of monojet production can then be found by integrating these cross sections with appropriate parton distributions.

The processes $q \bar{q} \rightarrow g Z^{0}, q g \rightarrow q Z^{0}$, followed by an invisible decay of the $Z^{0}$, give an irreducible physics background to $G$ production. We will refer to this process as the 'SM background', and we will estimate the observability of our signal by comparing its cross section to that of this reaction. There are other important background sources from mismeasured jets and $W$ production with forward leptons, but these backgrounds decrease sharply as the lower bound on missing $E_{T}$ is increased. Unlike the case of $e^{+} e^{-}$reactions, the detector does not measure the imbalance in longitudinal momentum, and there is not enough kinematic information from the single observed jet to exclude the kinematic region in which the $Z^{0}$ is on-shell. On the other hand, the parton center of mass energies available at the Tevatron are higher than those of LEP 2, and we have seen that the G signal increases rapidly with energy. It is therefore reasonable to look for the monojet signal as an excess above the SM cross section for on-shell $Z^{0}$ production. Though it is not so easy to compute the SM background rate accurately, this rate can be normalized to the corresponding process in which the $Z^{0}$ decays to a lepton pair.

The CDF collaboration has presented a bound on monojet production based on its first $4.7 \mathrm{pb}^{-1}$ of data in $p \bar{p}$ collisions at $\sqrt{s}=1.8 \mathrm{TeV}$ [14, 15]. This analysis searched for events with missing $E_{T}$ greater than $30 \mathrm{GeV}$ and one jet in the rapidity region $|y|<1.2$. The result was consistent with the $Z^{0}$ background and can be represented as a limit on the number of neutrinos into which the $Z^{0}$ decays: $N_{\nu}<5.0$ (95\% confidence). We convert this to a limit on $G$ production by the comparing the cross sections for the $G$ signal and the SM process, computed in the same framework. For simplicity, we carry out the calculations of both signal and background at the leading order in QCD, using the CTEQ4 lowest-order (set 3) structure functions [16]. We find a SM background cross section of $37 \mathrm{pb}$ for the cuts listed above, and, for $n=2$, a signal cross section of $20 \mathrm{pb} / M^{4}$. This implies a limit $R<1.2$ mm, $M>750 \mathrm{GeV}$. Limits for higher values of $n$ are given in Table 1. In Figure 2, we show the missing $E_{T}$ spectrum of the signal and background processes.

It is advantageous to make a tighter cut on missing $E_{T}$ to remove the backgrounds from mismeasured jets which were a problem for the CDF analysis [14]. Integrating the signal and background rates over the region with missing $E_{T}>60 \mathrm{GeV}$ and jet rapidity $|y|<2.4$, we find a SM background cross section for $\mathrm{Z}$ production of $10 \mathrm{pb}$, and signal cross sections 


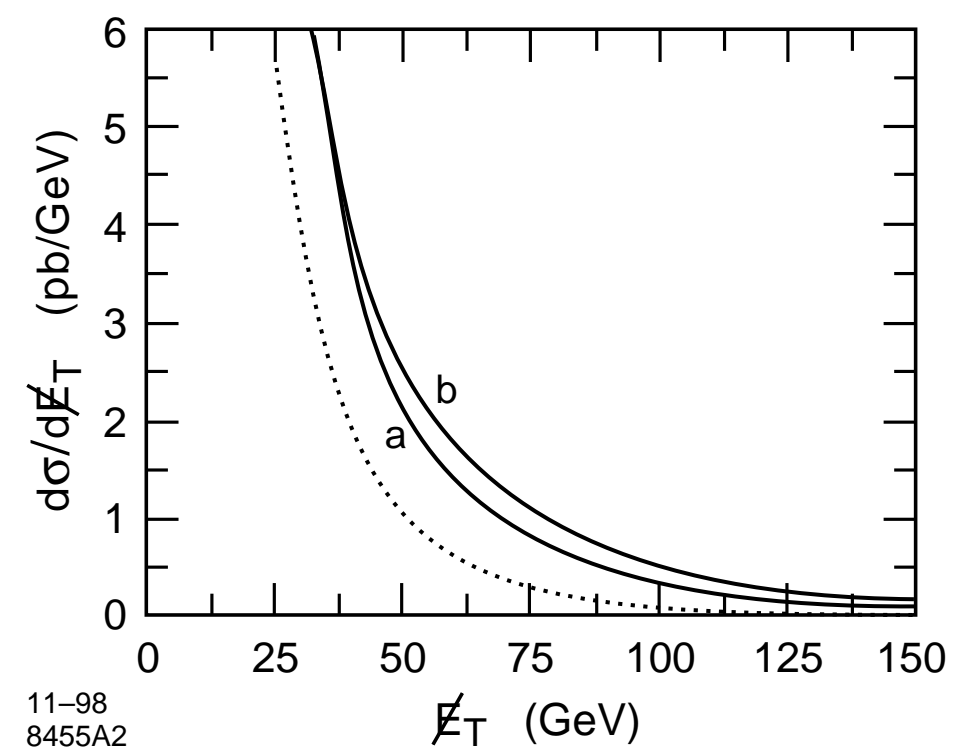

Figure 2: Spectrum of missing energy in events with one jet, computed for $p \bar{p}$ collisions at $\sqrt{s}=1.8 \mathrm{TeV}$, with a rapidity cut $|y|<2.4$. The dotted curve is the Standard Model expectation. The solid curves show the additional cross section expected in the model of ref. [4] with (a) $n=2, M=750 \mathrm{GeV}$, (b) $n=6, M=610 \mathrm{GeV}$.

in the ratios

$$
S / B=0.85 / M^{4}, 0.15 / M^{6}, 0.052 / M^{8}
$$

for $n=2,4,6$ and $M$ in TeV. Assuming that this measurement can be performed with $20 \%$ accuracy, and that the value to be found agrees with the SM, we find the potential limits on $R$ and $M$ listed in the third line of Table 1 .

Hadron-hadron collisions will be studied at higher energy at the CERN LHC. At the LHC, most collisions are between gluons, since the gluon structure functions rise rapidly at low $x$. This suppresses the SM contribution, since gluon-gluon collisions cannot lead to $Z^{0}$ production at the leader order in $\alpha_{s}$. However, we find that the most important contributions to $\mathrm{G}$ production also involve quarks, since the enhancement of the cross section at high energy partially compensates the falloff of the structure functions. Repeating the analysis leading to (12) at the LHC energy of $14 \mathrm{TeV}$ using the kinematic cuts $E_{T}>200 \mathrm{GeV},|y|<5$, we find a SM background cross section of $11 \mathrm{pb}$ and signal cross sections in the ratios

$$
S / B=110 / M^{4}, 420 / M^{6}, 3600 / M^{8},
$$

for $n=2,4,6$ and $M$ in TeV. With the same assumptions as for (12), we find the potential limits listed in the last line of Table 1. It is important to note that, in the case $n=6$, the dominant parton-parton center of mass energies are comparable to the quoted limit on $M$, so the effective coupling (2) might not be appropriate for this case. 


\begin{tabular}{ll|r|r|r} 
Collider & & $\mathrm{R} / \mathrm{M}(n=2)$ & $\mathrm{R} / \mathrm{M}(n=4)$ & $\mathrm{R} / \mathrm{M}(n=6)$ \\
\hline \hline Present: & LEP 2 & $4.8 \times 10^{-2} / 1200$ & $1.9 \times 10^{-9} / 730$ & $6.9 \times 10^{-12} / 520$ \\
\hline & Tevatron & $11.0 \times 10^{-2} / 750$ & $2.4 \times 10^{-9} / 610$ & $5.8 \times 10^{-12} / 610$ \\
\hline \hline Future: & Tevatron & $3.9 \times 10^{-2} / 1300$ & $1.4 \times 10^{-9} / 900$ & $4.0 \times 10^{-12} / 810$ \\
\hline & LC & $1.2 \times 10^{-3} / 7700$ & $1.2 \times 10^{-10} / 4500$ & $6.5 \times 10^{-13} / 3100$ \\
\hline LHC & $3.4 \times 10^{-3} / 4500$ & $1.9 \times 10^{-10} / 3400$ & $6.1 \times 10^{-13} / 3300$ \\
\hline
\end{tabular}

Table 1: Current and future sensitivities to large extra dimensions, expressed as 95\% confidence limits on the size of extra dimensions $R$ (in $\mathrm{cm}$ ) and the effective Planck scale $M$ (in $\mathrm{GeV})$. The assumptions of each analysis are explained in the text.

Conclusions. In this paper, we have shown that high-energy collider searches for events with missing energy and transverse momentum provide a relevant, model-independent test of theories with large extra space dimensions. Current high-energy experiments at $e^{+} e^{-}$and $p \bar{p}$ colliders already place the strongest direct constraints on these theories. Higher-energy experiments may place much stronger constraints. Or, more optimistically, they may allow us to observe an excess of missing-energy events above the SM expectation, providing direct evidence for this remarkable extension of our conception of the universe.

We are grateful to Nima Arkani-Hamed for suggesting this project and encouraging us along the way, and to Michael Barnett, Savas Dimopoulos, Lance Dixon, JoAnne Hewett, Ian Hinchliffe, Teruki Kamon, and Joseph Lykken for helpful discussions. This work was supported by the Department of Energy under contract DE-AC03-76SF00515. As this paper was being completed, we received a paper by Giudice, Rattazzi, and Wells that carries out an analysis very similar to the one presented here [17]. 


\section{References}

[1] I. Antoniadis, Phys. Lett. B 246, 377 (1990).

[2] This idea has recently been developed further by J. Lykken, Phys. Rev. D 54, 3693 (1996), hep-th/9603133, and by K. Dienes, E. Dudas, and T. Ghergetta, Phys. Lett. B 436, 55 (1998), hep-ph/9803466.

[3] R. Sundrum, hep-ph/9708329.

[4] N. Arkani-Hamed, S. Dimopoulos, and G. Dvali, Phys. Lett. B 429, 263 (1998), hepph/9803315; I. Antoniadis, N. Arkani-Hamed, S. Dimopoulos, and G. Dvali, Phys. Lett. B 436, 257 (1998), hep-ph/9804398; N. Arkani-Hamed, S. Dimopoulos, and G. Dvali, hep-ph/9807344.

[5] G. Shiu and S.-H. H. Tye, Phys. Rev. D 58, 106007 (1998), hep-th/9805157; Z. Kakushadze and S.-H. H. Tye, hep-th/9809147.

[6] The generality of this result is explained carefully in R. Sundrum, hep-ph/9805471.

[7] V. P. Mitrofanov and O. I. Ponomareva, Sov. Phys. JETP 67, 1963 (1988).

[8] Macroscopic bounds on modifications of gravity have recently been reviewed by J. C. Long, H. W. Chan, and J. C. Price, hep-ph/9805217.

[9] As $m^{2} \rightarrow 0$, this expression has a smooth limit in which only the helicity states $\gamma(-) G(+2)$ and $\gamma(+) G(-2)$ are produced.

[10] F. A. Berends, G. J. H. Burgers, C. Mana, M. Martinez, and W. L. van Neerven, Nucl. Phys. B 301, 583 (1988).

[11] R. Barate, et al. (ALEPH Collaboraton), Phys. Lett. B 429, 201 (1998).

[12] G. Abbiendi, et al. (OPAL Collaboration), hep-ex/9810021.

[13] As $m^{2} \rightarrow 0$, this expression has a smooth limit in which the only nonzero helicity amplitudes are those related by crossing to $g(+) g(+) g(-) \rightarrow G(+2)$ and $g(-) g(-) g(+) \rightarrow$ $G(-2)$. Though it is not so obvious in this representation, the squared matrix element contributing to (11) is symmetric under permutations of $s, t$, and $u$.

[14] R. Markeloff (CDF Collaboration), in The Fermilab Meeting, DPF '92, C. H. Albright, P. H. Kasper, R. Raja, and J. Yoh, eds. (World Scientific, Singapore, 1993); R. Markeloff, University of Wisconsin Ph. D. thesis, 1992.

[15] Later publications from the CDF and D0 experiments on searches for missing $E_{T}$ have required in addition the presence of at least 3 jets; see, for example M. Chertok, FERMILAB-Conf-98/156-E, to appear in the Proceedings of the 33rd Rencontres de Moriond: QCD and High Energy Hadronic Interactions, J. Tran Thanh Van, ed. 
[16] H. L. Lai, et al. (CTEQ Collaboration), Phys. Rev. D 55, 1280 (1997), hep-ph/9606399.

[17] G. F. Giudice, R. Rattazzi, and J. D. Wells, hep-ph/9811291. 